\title{
Analysis of the self-imaging effect in plasmonic multimode waveguides
}

\author{
André G. Edelmann, Stefan F. Helfert, ${ }^{*}$ and Jürgen Jahns \\ Optical Information Technology, FernUniversität in Hagen, Universitätsstrasse 27/PRG, 58084 Hagen, Germany \\ *Corresponding author: stefan.helfert@ fernuni-hagen.de \\ Received 16 July 2009; revised 28 September 2009; accepted 2 October 2009; \\ posted 2 October 2009 (Doc. ID 114364); published 29 October 2009
}

\begin{abstract}
We present studies on the propagation of plasmon waves in metallic multimode waveguides surrounded by a dielectric medium. The permittivity of the metal was determined by a Drude model. The propagation was simulated by the method of lines. The propagating field exhibited the well-known self-imaging phenomenon known as the Talbot effect. The metallic waveguides are lossy. The influence of various parameters on the losses was examined. By a suitable choice of parameters, propagation distances of several Talbot periods are possible. Our investigation also includes simulations for the propagation of eigenmodes of the waveguides and results for the calculation of the effective index. (C) 2010 Optical Society of America

OCIS codes: $\quad 050.1960,070.6760,130.2790,130.3120,230.7370,240.6680$.
\end{abstract}

\section{Introduction}

The field of plasmonics has emerged as a new area in which electronics and optics merge. A plasmon is an electromagnetic wave that propagates at optical frequencies along the surface of a nanoscale metallic wire [1]. Plasmonic waveguides offer the potential for miniaturization of all-optical circuits; probably better than optical high-index dielectric waveguides and similar to photonic crystal waveguides. As the main limitation of plasmonics one must consider the fact that, as a result of dissipation, only short transmission distances are possible, at least with current approaches. The hope is to use negative-index materials in the future so that long transmission distances become feasible. This would open the door to an integrated photonic-electronic circuitry. At the moment, a strong interest exists in modeling and simulation of plasmonic devices. The specific challenge in dealing with plasmonic propagation in comparison with more conventional optical waveguides lies in the fact that one has to deal with large index contrasts and that the material parameters (here the

0003-6935/10/0700A1-10\$15.00/0

(C) 2010 Optical Society of America permittivity) can be complex with a large negative real part.

In the past, several plasmonic devices have been studied. In particular we would like to mention plasmonic sensors (see, e.g., [2,3]) and plasmonic lenses (see, e.g., $[4,5]$ ). Here we consider another phenomenon that is well known from diffraction optics, namely, the self-imaging effect. For plasmonic wave fields, self-imaging was the topic of an earlier investigation [6]. However, in that case, the considered structure was unbounded in the lateral direction. Here, we go beyond that case by analyzing wave propagation in a plasmonic multimode waveguide in which lateral bounding is essential. The fact that self-imaging can occur in multimode waveguide structures is known from conventional dielectric waveguides as we will explain later.

Here, our goal is threefold: first, we analyze the propagation of plasmonic multimode fields and study plasmonic self-imaging to understand the physical situation by comparing the results with the wellknown phenomenon from optics. Second, we adapt our simulation tools to this interesting situation. The challenge consists in the fact that here we must deal with large dielectric index contrasts and complex material parameters. As a tool for the simulation, 
we use the method of lines (MoL) [7,8], which is a semianalytical simulation procedure that is suitable to solve partial differential equations. In particular, the MoL has been applied to analyze waveguide structures at microwave and optical frequencies. In addition, we would like to mention that the MoL has also been used to solve the heat equation in laser structures [9]. Here we wish to see how well our simulation tool is suited to simulate such lossy structures or if improvements are required. Finally, the self-imaging effect in metallic multimode waveguides might have interesting applications in later integrated plasmonic devices. As a specific application of self-imaging in dielectric structures, so-called multimode interference devices [10] have been demonstrated as integrated beam splitters. Such beam splitters would be required to implement signal fanout in integrated plasmonic circuits.

In Section 2 we give a brief review of self-imaging in multimode waveguides and explain the principles of the MoL. Section 3 shows our examined structure. Section 4 contains the focus of our study, which is the simulation results. In particular, we examine the influence of various parameters on the losses and study the wave propagation in plasmonic waveguides. We conclude with a summary in Section $\underline{5}$.

\section{Theory}

\section{A. Self-Imaging in Multimode Waveguides}

Self-imaging means that a wave field repeats itself after a certain propagation distance. A special case of self-imaging is the Talbot effect [11], which is well known from optical near-field diffraction. The Talbot effect occurs for paraxial, monochromatic wave fields with a lateral period $p_{x}$. By suitable superposition of the modes the field repeats itself after a distance of $z_{T}=2 p_{x}^{2} / \lambda$, with $\lambda$ being the wavelength, i.e.,

$$
u(x, z)=u\left(x, z+z_{T}\right) e^{j \phi},
$$

where $\phi$ is a suitable phase factor that is identical for all modes. In free space, for example, it can be determined as $k_{0} z_{T}$. Here, and in what follows, we use $x$ as a transverse coordinate and $z$ as a longitudinal coordinate. For the principal explanations in this section we restrict ourselves to two-dimensional (2-D) structures. However, in later sections we will look at the three-dimensional (3-D) case.

The Talbot effect can be observed in free space and in multimode waveguides. In the free-space optical case, the lateral periodicity is imposed on the wave field by a grating of period $p_{x}$. The free-space optical Talbot effect has been widely analyzed and suggested for applications in interferometry [12] and for temporal filtering [13]. As mentioned before, the plasmonic analog was described in [6].

Self-imaging in multimode waveguides was described as early as the 1970s [14,15] and suggested for beam-splitting applications in the 1990s [10]. In a waveguide, a virtual lateral periodicity exists that is due to reflecting sidewalls. This virtual periodicity accounts for the discrete modal spectrum of the wave field. The periodicity is not exact since, for different modes, the lateral period is slightly different. This is caused by a different extension of the modal field into the area of the cladding for different modes (see Fig. 1). However, as shown in [16], one can describe the virtual periodicity approximately by $p_{x} \approx 2 w$.

The theory of self-imaging in multimode waveguides was described in detail in [10]. Therefore, here we restrict ourselves to a few remarks. The propagation constants of the guided modes in multimode waveguides have a (nearly) quadratic behavior with respect to the mode number. This can be seen in Fig. 1(b) where we show the normalized propagation constant (or effective index $n_{\text {eff }}=k_{z} / k_{0}$ ) of the modes for the waveguide on the top. The self-imaging length for an arbitrary input field can be determined from the difference in propagation constants of the lowestorder modes [10]. Depending on the shape of the input field, one can also obtain images at fractions of the self-imaging distance.

\section{B. Method of Lines}

For numerical analysis of the metallic structures, we use the MoL $[7,8]$. The MoL has been successfully applied to simulate waveguide devices in the optical and microwave domains. The interested reader is referred to two overview articles $[17,18]$. The analysis of plasmonic devices by different numerical methods including the MoL was presented in [19-21]. Since we perform full 3-D calculations here, we also

(a)

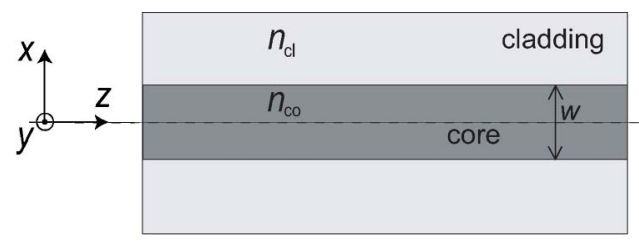

(b)

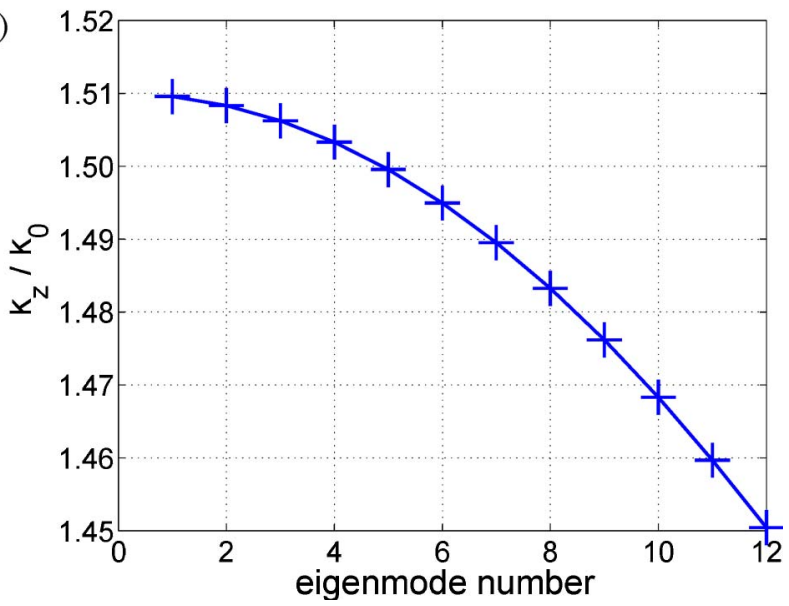

Fig. 1. (Color online) (a) Dielectric multimode waveguide structure and (b) normalized propagation constants of the eigenmodes for $w=20 \mu \mathrm{m}$, wavelength $\lambda=1.5 \mu \mathrm{m}, n_{\mathrm{co}}=1.52$, and $n_{\mathrm{cl}}=1.45$. This waveguide was studied in [16]; the Talbot distance was determined as $z_{T}=3600 \mu \mathrm{m}$. 
mention [22,23], because these papers showed how the numerical effort can be kept moderate when analyzing such 3-D structures. Here, we present just a brief summary of the MoL, which is a semianalytic technique that operates in the frequency domain. In our computations, we assume a time dependency according to $\exp \{j w t\}$.

To apply the MoL we start by dividing the circuit into sections in which the material parameters do not depend on the direction of the wave propagation (in our case the $z$ direction). From Maxwell's equation we can then derive coupled partial differential equations for the transverse components of the electric or magnetic field (here we proceed with the magnetic field) in the following form [24]:

$$
\frac{\partial^{2}}{\partial \bar{z}^{2}} \vec{H}_{t}-Q_{H} \vec{H}_{t}=\overrightarrow{0} \quad \text { with } \vec{H}_{t}=\left(\begin{array}{c}
\tilde{H}_{y} \\
-\tilde{H}_{x}
\end{array}\right) .
$$

Operator $Q_{H}$ contains derivatives with respect to the transverse coordinates (i.e., $x, y$ ) and the material parameters $\epsilon_{r}(x, y)$ and $\mu_{r}(x, y)$; see, e.g., [18]). Further, the overlined coordinates are normalized with the free-space wavenumber $k_{0}$ (e.g., $\bar{z}=k_{0} z$ ). Equation (2) is a coupled partial differential equation system. To solve it, we discretize the fields in the cross section and approximate the derivatives with respect to these coordinates with finite differences. After putting all the discretized fields into a supervector and combining the finite differences into a matrix, we obtain a system of ordinary differential equations (ODEs):

$$
\frac{\partial^{2}}{\partial \bar{z}^{2}} \hat{\mathbf{H}}-\hat{\boldsymbol{Q}}_{H} \hat{\mathbf{H}}=\mathbf{0} .
$$

As known from mathematics, such ODEs can be solved with a transformation to the principal axes, i.e., determining the eigenvalues and eigenvectors of $\hat{\boldsymbol{Q}}_{H}$ :

$$
\hat{\boldsymbol{Q}}_{H}=\hat{\boldsymbol{T}}_{H}^{-1} \Gamma^{2} \hat{\boldsymbol{T}}_{H} .
$$

Now, by transforming the field according to

$$
\hat{\mathbf{H}}=\hat{\boldsymbol{T}}_{H} \hat{\mathbf{H}}
$$

and replacing $\hat{\boldsymbol{Q}}_{H}$ with the expression given in Eq. (4), we obtain a system of decoupled differential equations whose solution can be easily given as

$$
\hat{\mathbf{H}}(z)=e^{-\Gamma \bar{z}} \hat{\mathbf{H}}_{f}(0)+e^{\Gamma \bar{z}} \hat{\mathbf{H}}_{b}(0) .
$$

The subscripts $f$ and $b$ mean forward and backward, respectively. Now, computing the eigenvalues and eigenvectors in Eq. (3) corresponds to a determination of the eigenmodes in the examined section. Each column of the eigenvector matrix $\hat{\boldsymbol{T}}_{H}$ represents the field distribution of these modes; the square roots of the eigenvalues represent the propagation constant.
For the propagation constant of the $k$ th mode we can write

$$
\Gamma_{k}=\bar{\alpha}_{k}+j \bar{\beta}_{k}
$$

As previously (with the coordinates), the overbar indicates a normalization with $k_{0}$. The denormalized values are obtained by multiplication with $k_{0}(\alpha=$ $\left.k_{0} \bar{\alpha}, \beta=k_{0} \bar{\beta}\right)$. Instead of $\Gamma$ we often use the effective index to describe the propagation of the eigenmodes. Their relation is simply given by

$$
n_{\mathrm{eff}}=\bar{\beta}-j \bar{\alpha}=-j \Gamma .
$$

As is known, the imaginary part of the effective index $(\bar{\alpha})$ describes the losses. For guided modes in dielectric waveguides it is zero. Then $\bar{\beta}$ is just the effective index of the structure.

The values $\overline{\mathbf{H}}_{f, b}$ in Eq. (ㅁ) are the complex amplitudes of the forward and backward propagating modes. Equation (5) shows how a particular field distribution of these eigenmodes is put together. Equation (6) is the general solution for the fields in a homogeneous section (homogeneous section with respect to the $z$ coordinate). Often we have more complex devices that consist of more than one homogeneous section. In this case we must apply Eqs. (2) (6) in each of them. Next we must assure the continuity of the transverse electric and magnetic field components. Therefore, besides the magnetic fields, we must also determine the transverse electric fields $\bar{E}$. From Maxwell's equations the following relation can be derived [24]:

$$
\frac{d}{d \bar{z}} \hat{E}=-j \hat{R}_{H} \hat{H}(z) .
$$

Similar to $\hat{\boldsymbol{Q}}_{H}$ the matrix $\hat{\boldsymbol{R}}_{H}$ contains finite difference approximations of derivatives with respect to $x$ and $y$ and the material parameters (for details see, e.g., [8]). Since the solution in the $z$ direction is given in an analytic form, we can easily determine the electric field from this equation as

$$
\hat{\boldsymbol{E}}(z)=\hat{\boldsymbol{T}}_{E}\left(\hat{\overline{\mathbf{H}}}_{f}(z)-\hat{\overline{\mathbf{H}}}_{b}(z)\right)
$$

with $\hat{\boldsymbol{T}}_{E}=j \hat{\boldsymbol{R}}_{H} \hat{\boldsymbol{T}}_{H} \boldsymbol{\Gamma}^{-1}$. As said before, the overlined magnetic fields represent the amplitudes of the eigenmodes. From Eq. (10) we see that the electric fields are obtained by multiplying these amplitudes with the corresponding electric field distribution (given by $\hat{\boldsymbol{T}}_{E}$ ). By enforcing the continuity of the transverse fields at the interfaces and assuming suitable boundary conditions, we are able to analyze complete complicated circuits. All relevant physical phenomena can be included in the algorithm. Obviously, reflections at interfaces are included, since we consider forward and backward propagating modes. To model radiation of the fields we introduce absorbing boundary conditions into the finite difference expressions 
[25]. Loss and gain of the materials are considered by complex numbers for the material parameters.

After these introductory remarks with regard to the MoL, we wish to emphasize one of the relevant aspects of this work. For the plasmonic devices considered here, the important question is whether our simulation method had to be further extended to handle the large contrast differences in material parameters and negative real values of the permittivity. As we show in the following, at least for the devices examined here, the MoL proved to be a completely adequate tool.

\section{Model of the Examined Structure}

To study the properties of the plasmon wave propagation we use the structure shown in Fig. 2(a) as the basis. It consists of a metal core and a surrounding dielectric material. In general, this material could, for example, differ above and below the metal. Here, we just examined the symmetric case. The permittivity of the metal was determined from a Drude model:

$$
\epsilon_{m}=\epsilon_{\infty}-\frac{\omega_{p}^{2}}{\omega^{2}-j \gamma \omega} .
$$

Note that, because of the time dependency according to $\exp \{j \omega t\}$, the imaginary part of $\epsilon_{m}$ is less than zero in the case of lossy material, which is why there is a negative sign in front of $\gamma$ in Eq. (11). We would like to

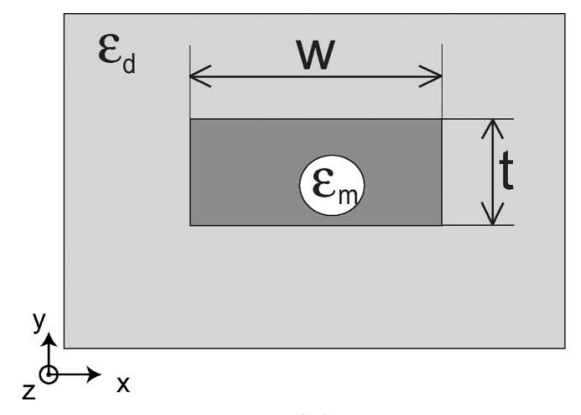

(a)

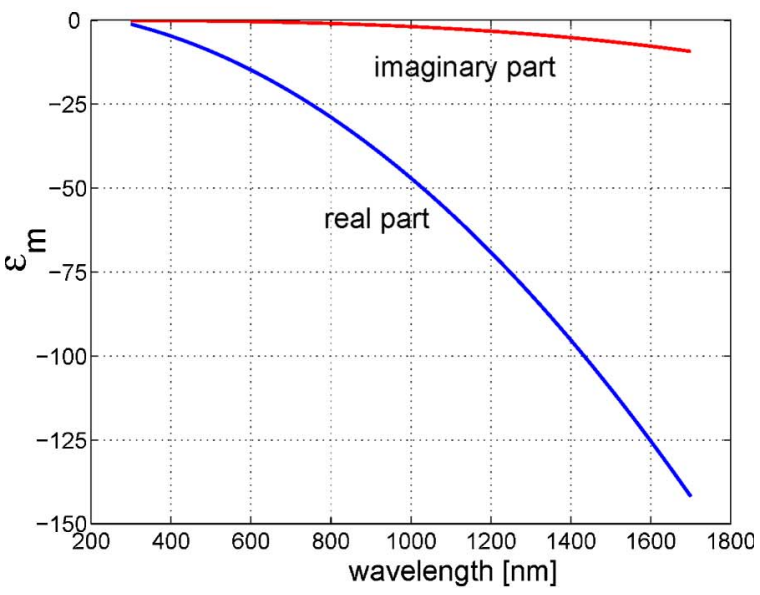

(b)

Fig. 2. (Color online) (a) Examined metallic waveguide structure and (b) frequency dependency of $\epsilon_{m}$ (permittivity of metal). point out that slightly different values for the parameters can be found in the literature. Here we use the following parameters taken from [20]: $\epsilon_{\infty}=$ $3.36174, \omega_{p}=1.3388 \times 10^{16} \mathrm{~s}^{-1}$, and $\gamma=\overline{7} .07592 \times$ $10^{13} \mathrm{~s}^{-1} . \epsilon_{r}$ as a function of the wavelength in the optical regime is presented in Fig. 2(b). We see that the real part as well as the imaginary part is negative and that their absolute value increases with increasing wavelength. In the following simulations, several of the parameters in Fig. 2(a) will be varied to evaluate their influence on the waveguide losses.

\section{A. Eigenmodes of Multimode Waveguides}

As described in Subsection 2.A, more than two guided modes with different $k$ vectors are required to obtain the self-imaging effect. Therefore, we start with a comparison of the eigenmodes in dielectric and metallic waveguides. The dielectric structure we use for comparison is similar to the metallic one shown in Fig. 2. Instead of a metallic core with the permittivity $\epsilon_{m}$ however, the core consists of a dielectric. Furthermore, height $t$ is different.

As is usual in optics, we consider materials with $\mu_{r}=1$. The wavelength was chosen to be $\lambda=$ $600 \mathrm{~nm}$. The other parameters are: waveguide width $w=2 \mu \mathrm{m}$ and permittivity of the surrounding material $\epsilon_{d}=4$. For the dielectric waveguide we chose the value of $2.2^{2}=4.84$ for the relative permittivity in the core and the height $t=0.5 \mu \mathrm{m}$. For comparison, for the metallic waveguide we use $\epsilon_{m}=-14.7897-$ $j 0.4088$ and $t=0.1 \mu \mathrm{m}$.

The magnetic field distributions of the significant eigenmodes are shown in Fig. 3. Due to $w \gg t$ for the metal structure, the interesting polarization for plasmon waves is the one in which $H_{x}$ is the main component of the magnetic field, which is usually referred to as TM polarization. Therefore, we examine only the lowest-order TM modes in the case of dielectric waveguides.

For metallic waveguide, the fields represent two sets of modes. One set is symmetric around the $y$ axis, the second set is antisymmetric. For the chosen height of $t=0.1 \mu \mathrm{m}$, there is no obvious difference in terms of absolute value. However, as we will see later, the propagation constants are different.

Let us now compare the fields shown in Fig. 3. As is well known, waveguiding in a dielectric waveguide is due to total internal reflection. Therefore the field is concentrated in the core as we see in the graphs at left in Fig. 3. On the other hand, for a metallic waveguide, the propagating field represents a surface wave with the highest amplitude concentrated at the interface between the metal and the dielectric. Besides these differences, there are also some similarities, which become obvious by looking at the maximum field amplitudes; see Fig. 4. For the dielectric waveguide, this occurs at the center of the waveguide; for the metallic waveguide, this occurs at the surface. Both amplitude distributions look similar. In particular, a multimodal field occurs in both cases (which is a necessary condition for self-imaging), 

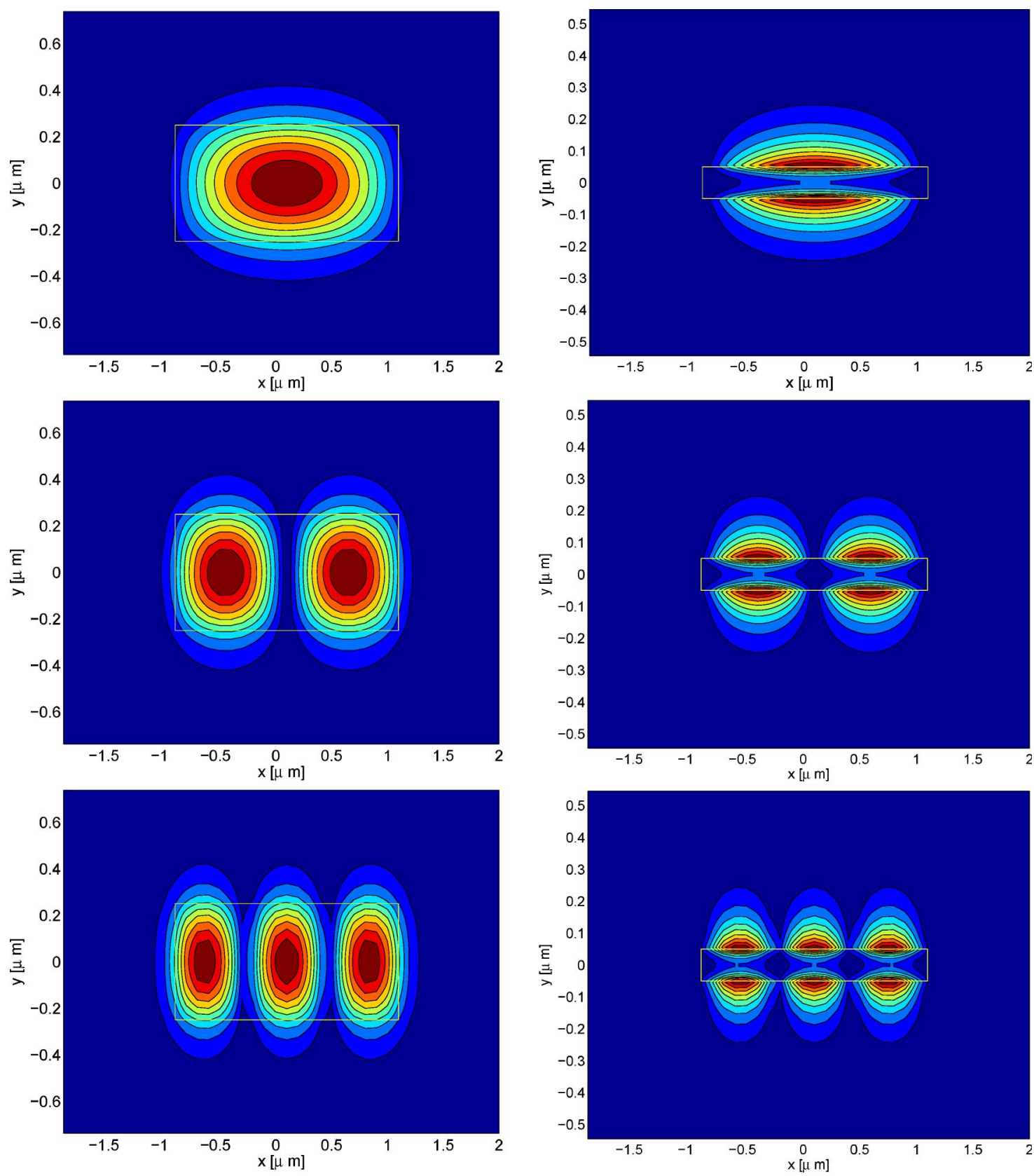

Fig. 3. (Color online) Magnetic field distribution $\left(H_{x}\right)$ of the eigenmodes in a dielectric (left) and a metallic waveguide (right).

and the maxima of different modes occur at nearly the same positions.

\section{Numerical Simulations}

\section{A. Losses in Metallic Waveguides}

Attenuation is a particular problem of current plasmonic devices. To analyze this in detail, we examine various geometric parameters of the waveguide to determine an optimal configuration.

In Fig. 5 we examined the normalized propagation constant of the eigenmodes as a function of height $t$ of the waveguide (the other parameters are the same as in Subection 3.A). Similar studies were already per- formed by other authors, see, e.g., $[2,26]$. As can be seen, the even and the odd modes behave completely differently. Both $\alpha$ and $\beta$ increase with decreasing height for the odd modes but decrease for the even modes. As shown in Fig. 5, the three even and the three odd modes, respectively, are nearly identical. This behavior can be understood with a simplified 2 -D model of the waveguide. For this purpose we examine a structure in which the metal layer extends to infinity in the horizontal direction $(w \rightarrow \infty$ hence, $\partial / \partial x=0)$. For this situation, we can derive analytical expressions for the propagation constant (or for the effective index), which can be achieved by determining the eigenmodes. The procedure is described in 

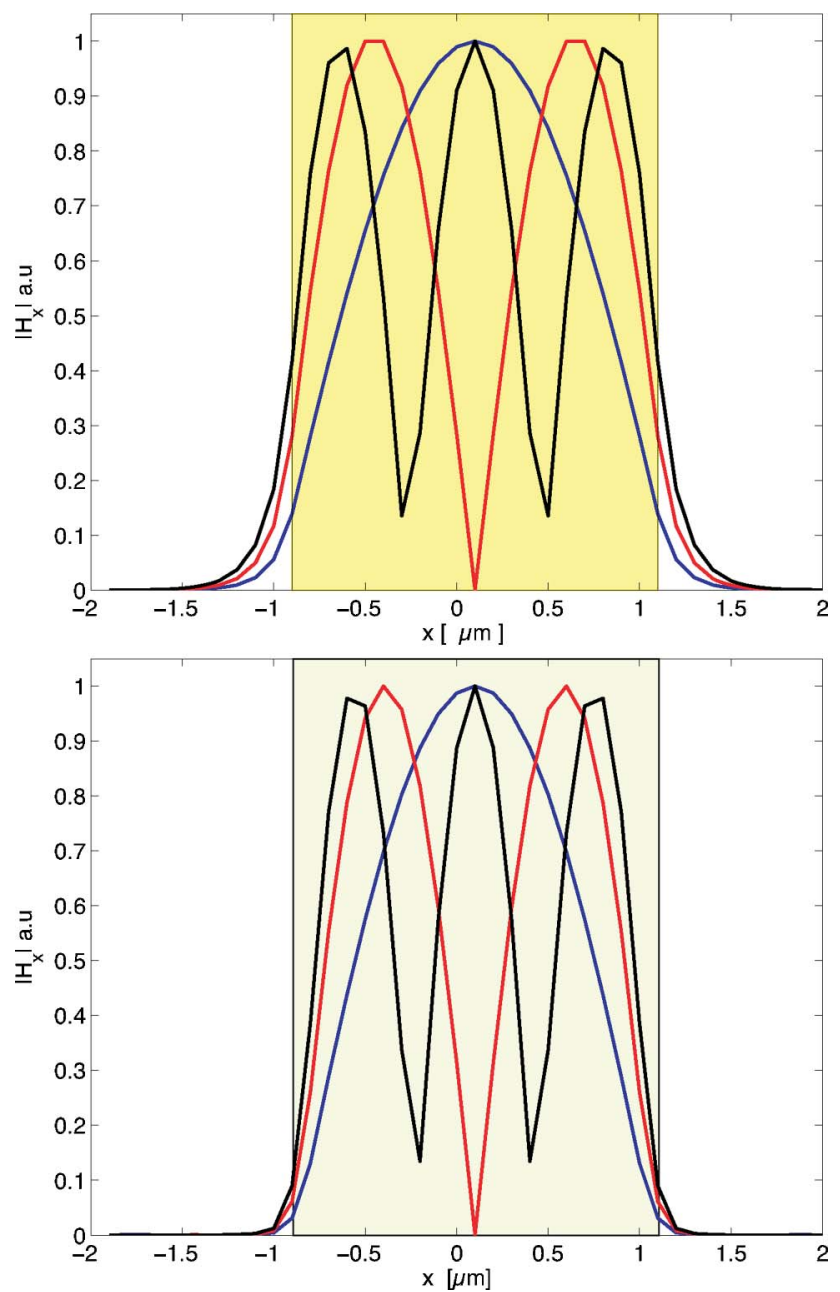

Fig. 4. (Color online) Magnetic field distribution $\left(H_{x}\right)$ in the center of a dielectric waveguide (top) and at the metal-dielectric surface (bottom).

basic textbooks. We end up with the following implicit expressions for the effective index:

$$
\begin{aligned}
& \tanh \left(\frac{k_{0} t}{2} \sqrt{n_{\mathrm{eff}}^{2}-\epsilon_{m}}\right)=-\frac{\epsilon_{m}}{\epsilon_{d}} \sqrt{\frac{n_{\mathrm{eff}}^{2}-\epsilon_{d}}{n_{\mathrm{eff}}^{2}-\epsilon_{m}}}(\text { even }), \\
& \operatorname{coth}\left(\frac{k_{0} t}{2} \sqrt{n_{\mathrm{eff}}^{2}-\epsilon_{m}}\right)=-\frac{\epsilon_{m}}{\epsilon_{d}} \sqrt{\frac{n_{\mathrm{eff}}^{2}-\epsilon_{d}}{n_{\mathrm{eff}}^{2}-\epsilon_{m}}}(\text { odd }) .
\end{aligned}
$$

Identical expressions, only in a slightly different form, were given in [27]. To examine them, we first consider thick layers. Then the fields on the two sides of the metal are decoupled. Mathematically, both hyperbolic functions (i.e., tanh and coth) approach the value of one in this case, and we can derive the well-known expression for plasmon waves of a bulk material:

$$
n_{\mathrm{eff}}=\sqrt{\frac{\epsilon_{d} \epsilon_{m}}{\epsilon_{d}+\epsilon_{m}}}
$$
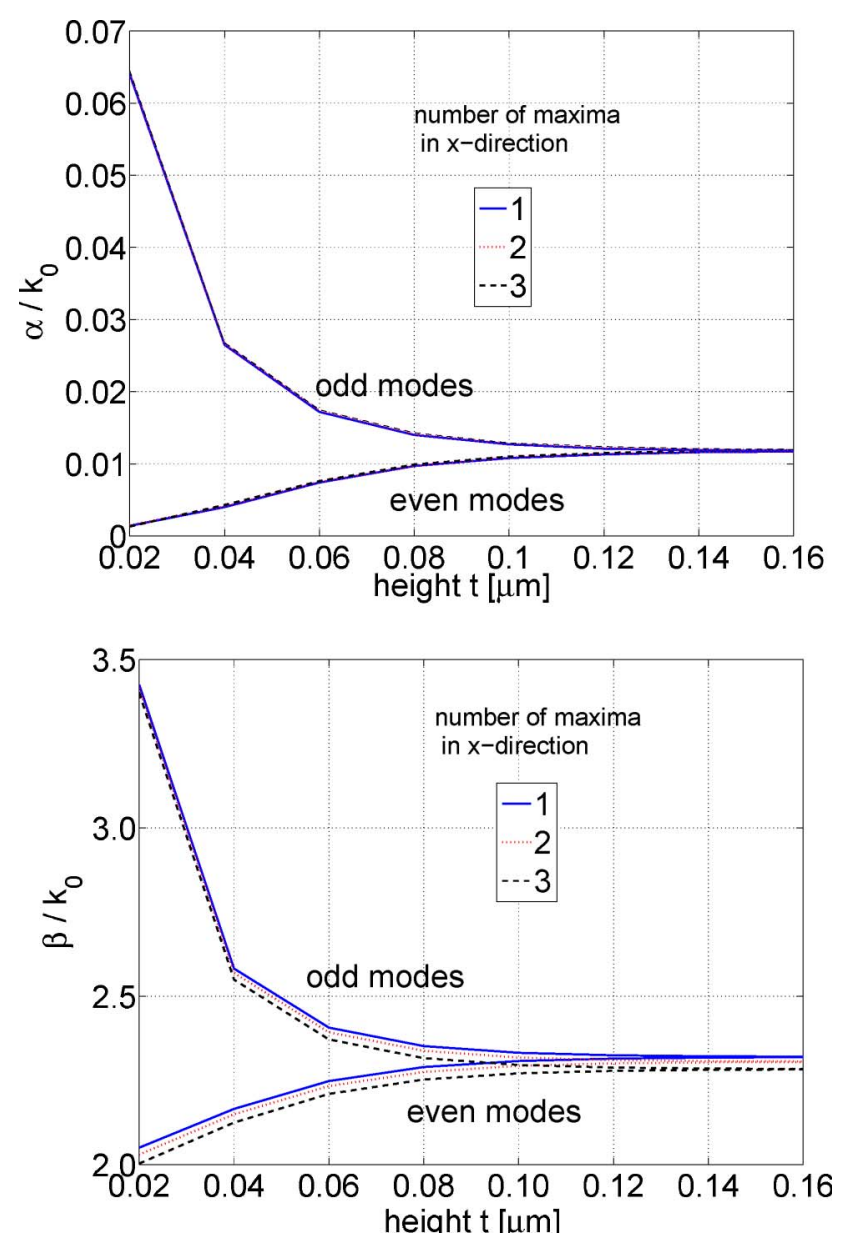

Fig. 5. (Color online) Normalized propagation constants of the first even and odd modes in the plasmonic waveguide: top, loss factor; bottom, phase factor; wavelength $\lambda=600 \mathrm{~nm}$.

From Eq. (14) it follows that the permittivities must fulfill the condition $\left|\mathscr{R}\left(\epsilon_{m}\right)\right|>\epsilon_{d}$ (see also, e.g., [1]). However, with regard to losses it is more interesting to examine the expressions in Eqs. (12) and (13) for small values of $t$. Here, significant differences occur between the even and the odd modes. For the even modes the tanh function on the left-hand side of Eq. (12) approaches zero for small values of $t$. Therefore, $n_{\text {eff }}^{2}$ must approach $\epsilon_{d}$ (i.e., the permittivity of the surrounding dielectric) on the right-hand side. If we assume that the dielectric has no (or only small) losses, the waveguide structure has low losses as well.

The situation is different for the odd mode whose effective index is determined by using Eq. (13). For a qualitative assessment, one can use a Laurent series expansion of the coth expression on the left-hand side of Eq. (13). Without going too much into the mathematical details, we mention that both the real part of $n_{\text {eff }}$ as well as its imaginary part (which represents the losses) approach infinity. Hence, although the losses of the even modes decrease with decreasing $t$, the losses of the odd mode increase at the same time. In Fig. $\underline{6}$ we present $n_{\text {eff }}$ in the complex plane. For large values of $t(t \rightarrow \infty)$ the even and odd modes 
start at the value given by Eq. (14). When $t$ decreases, the curves continue in opposite directions. As stated above, the effective index of the even mode approaches $\sqrt{\epsilon_{d}}$ of the surrounding medium, whereas the real and imaginary parts of $n_{\text {eff }}$ approach infinity for the odd modes.

As has been shown in Fig. 5, such behavior occurs not only in 2-D but also in 3-D structures, i.e., with finite width $w$. To design devices with low losses, the results suggest the use of structures with a small height of the metal. However, as we also see in Fig. 5, $\beta / k_{0}$ approaches the value of the refractive index of the surrounding dielectric material (which is 2 in our case). This has the consequence that the field reaches far into the surrounding medium, which necessitates the search for a compromise.

In Fig. 7 we see the effective index of the first odd plasmon mode as a function of the wavelength with the value of the surrounding permittivity as the parameter. (The curves for the even modes appear to be similar so are not shown here.) From Fig. 7 one can tell that the real and imaginary parts (an $\bar{d}$ therefore the losses) of $n_{\text {eff }}$ decrease with increasing wavelength. From the Drude model [Fig. 2(b)] we know that the losses in metal increase with the wavelength $\lambda$. These two results appear counterintuitive. However, it can be explained by the fact that the losses are not due only to dissipation in the metal. Rather, as can be seen from the following analysis, the absolute value of the permittivity is even more important to explain this behavior (see also [26]). This can be understood by examining the propagation constant for bulk metal in Eq. (14). For large wavelengths, not only the imaginary part of $\epsilon_{m}$ but also its real part and therefore the absolute value increase. Hence, for large wavelengths, $\epsilon_{d}$ can be neglected in the denominator of Eq. (14). Therefore, the expression simplifies to $n_{\text {eff }}=\sqrt{\epsilon_{d}}$. Since the surrounding dielectric material is assumed to be lossless, the structure is also lossless for large values

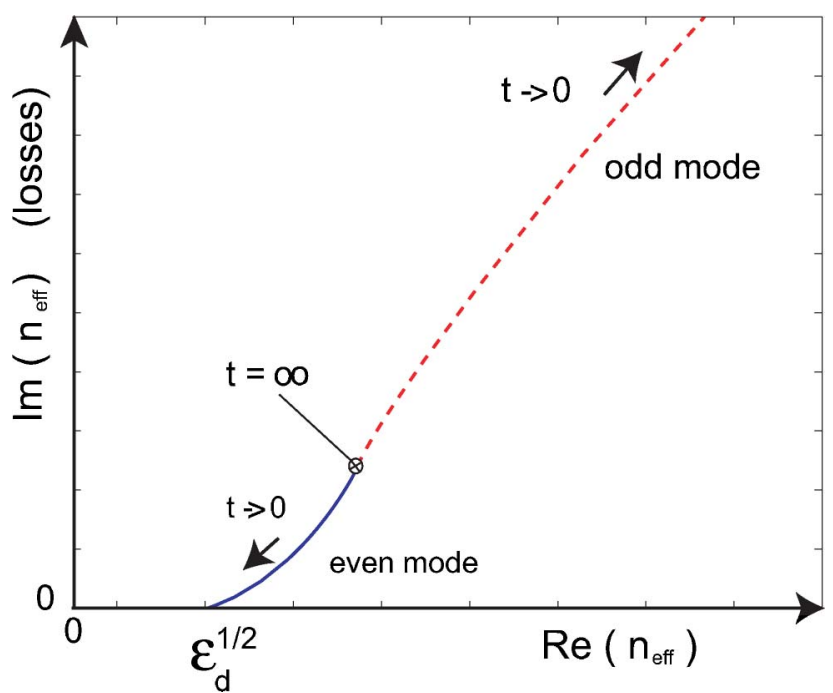

Fig. 6. (Color online) Effective index of the plasmon modes in a 2D waveguide.
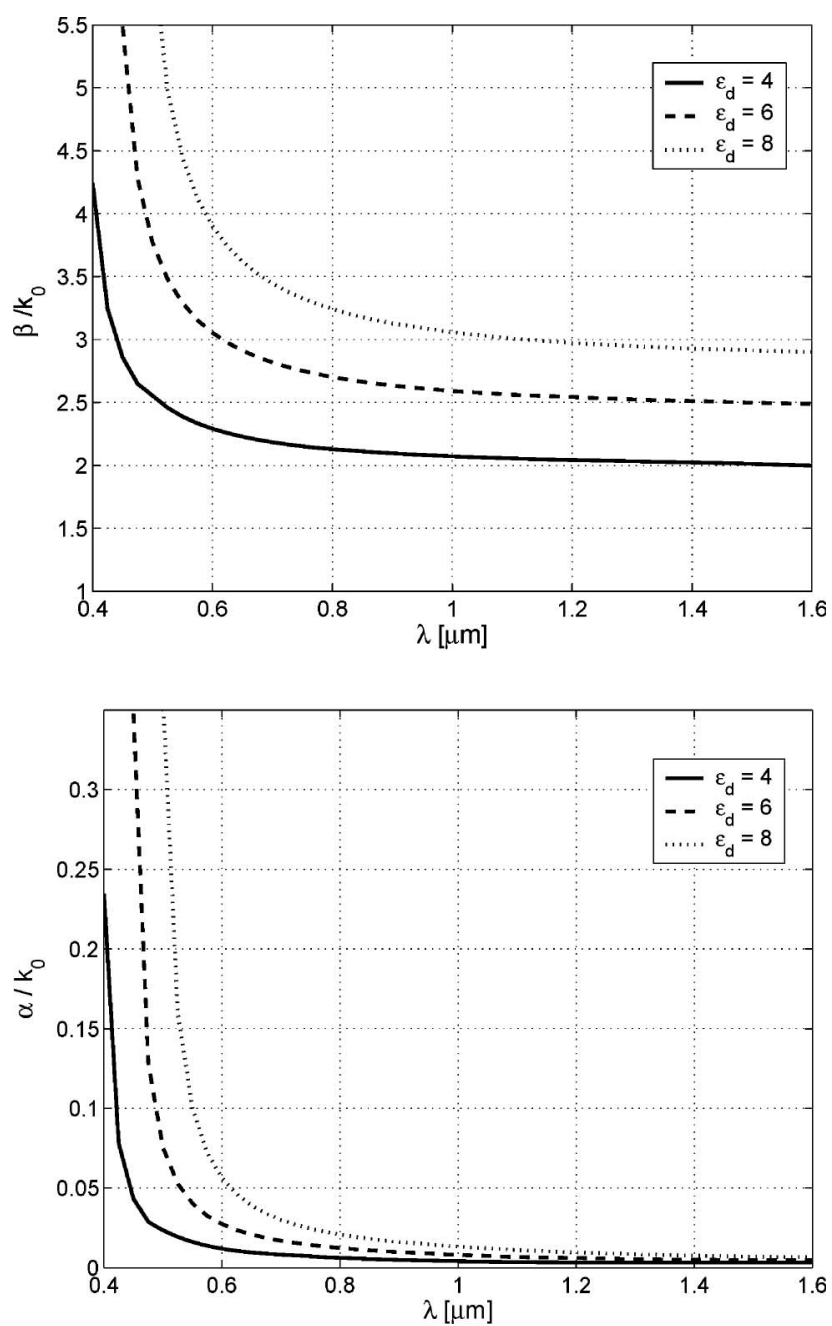

Fig. 7. Effective index of the first odd mode in a metallic waveguide (Fig. 2) as a function of the wavelength with the permittivity of the surrounding material as the parameter: height, $t=0.1 \mu \mathrm{m}$ and width, $w=2 \mu \mathrm{m}$.

of the $\left|\epsilon_{m}\right|$. This is the same reasoning we used before when we decreased height $t$ of the waveguide.

For a more quantitative prediction we take into account that, to have plasmonic waves, the condition $\left|\epsilon_{d}\right|<\left|\epsilon_{m}\right|$ must be fulfilled. This permits us to approximate $n_{\text {eff }}$ in Eq. (14) with a Taylor series:

$$
n_{\mathrm{eff}} \approx \sqrt{\epsilon_{d}}\left(1-\frac{\epsilon_{d}}{2 \epsilon_{m}}\right) .
$$

We now write the permittivity of the metal in the form $\epsilon_{m}=-\left|\epsilon_{\mathrm{re}}\right|-j\left|\epsilon_{\mathrm{im}}\right|$. After a few algebraic steps we find that we can write the following for $n_{\text {eff }}$ :

$$
n_{\mathrm{eff}}=\sqrt{\epsilon_{d}}+\frac{\epsilon_{d}^{3 / 2} \epsilon_{m}}{\left|\epsilon_{\mathrm{re}}\right|^{2}+\left|\epsilon_{\mathrm{im}}\right|^{2}}-j \epsilon_{d}^{3 / 2} \frac{1}{2} \frac{\left|\epsilon_{\mathrm{im}}\right|}{\left|\epsilon_{\mathrm{re}}\right|^{2}+\left|\epsilon_{\mathrm{im}}\right|} .
$$

To estimate the losses we examine the imaginary part of Eq. (16). The absolute value of the 


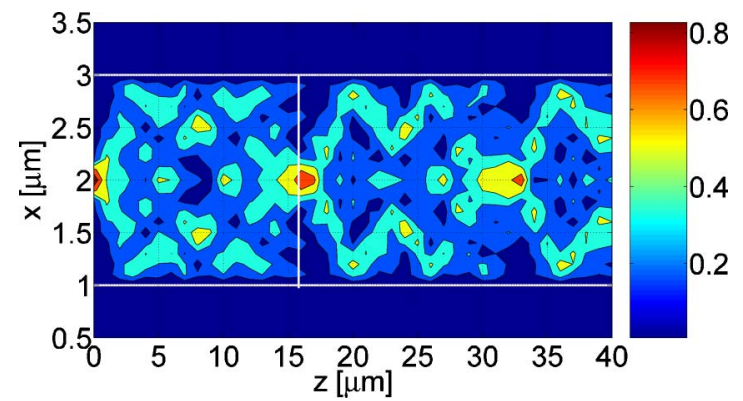

Fig. 8. (Color online) Field distribution of the $H_{x}$ component at the interface between the dielectric and the silver layer for $\lambda_{0}=0.6 \mu \mathrm{m}$; the imaginary part of $\epsilon_{m}$ was neglected.

permittivity of the metal is squared in the denominator, whereas the numerator contains $\left|\epsilon_{i m}\right|$ only linearly. Hence the complete term becomes small for large values of $\left|\epsilon_{m}\right|$ and increases for small values in agreement with the results we found. We can also see that the losses increase when the permittivity of the surrounding dielectric media increases, which is in agreement with the results found in Fig. 7. As we discovered when we examined the influence of height $t$ on the losses, the real part of the effective index approaches the refractive index of the surrounding material (leading to field distributions that reach far into the dielectric) for configurations with low losses.

\section{B. Simulation of Self-Imaging}

To prepare for the simulations presented in this subsection we introduce the self-imaging distance $L_{\mathrm{si}}$. We would like to point out the difference between the Talbot distance $z_{T}$ as used in Eq. (1) and the self-imaging distance $L_{\mathrm{si}}$. To understand the differences one must know that self-imaging can also occur at fractional distances of $z_{T}$. Particularly for symmetric excitation of the waveguide, self-imaging can occur at distances much less than $z_{T}$. The shortest distance at which self-imaging is observed is called $L_{\mathrm{si}}$. According to the theory given in [10], $L_{\mathrm{si}}$ for symmetrical excitation can be calculated as

$$
L_{\mathrm{si}}=\frac{3}{4} L_{\pi}=\frac{3}{8} \frac{\lambda_{0}}{\Delta \bar{\beta}} .
$$

Here $\Delta \bar{\beta}$ is the difference in normalized propagation constants of the two lowest-order modes. We now pre-

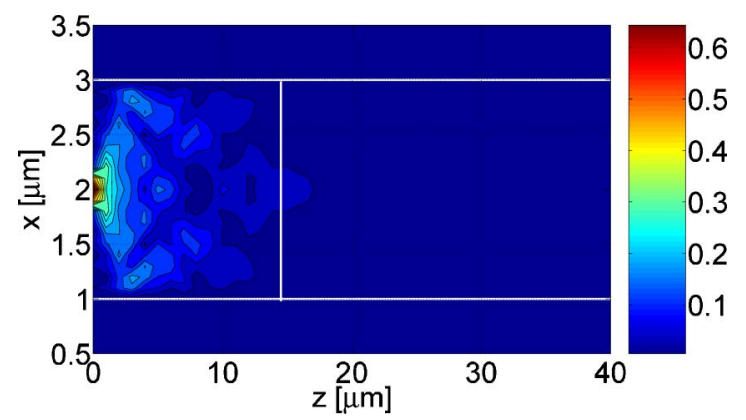

Fig. 9. (Color online) Field distribution of the $H_{x}$ component at the interface between the dielectric and the silver layer at $\lambda_{0}=0.6 \mu \mathrm{m}$; the influence of $\mathfrak{I}\left(\epsilon_{m}\right)$ is considered.

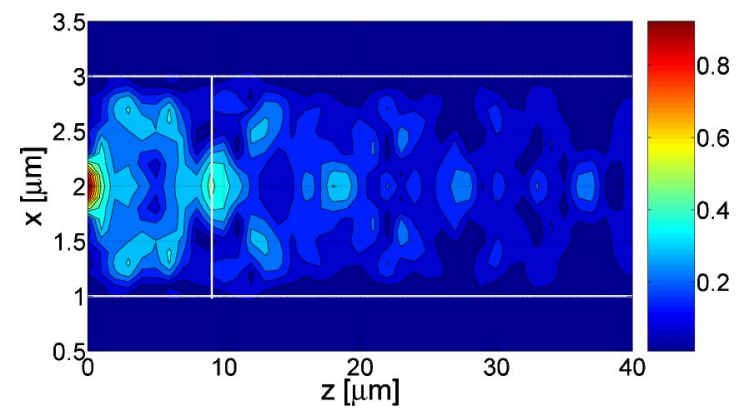

Fig. 10. (Color online) Field distribution of the $H_{x}$ component at the interface between the dielectric and the silver layer at $\lambda_{0}=0.9 \mu \mathrm{m}$; the influence of $\mathfrak{s}\left(\epsilon_{m}\right)$ is considered.

sent results for the wave propagation in the metallic structure shown in Fig. 2(a). First we used the following parameters: $\lambda_{0}=0.6 \mu \mathrm{m}, w=2 \mu \mathrm{m}, t=0.1 \mu \mathrm{m}$, and $\epsilon_{d}=4$. The value $\epsilon_{m}=-14.7897-j 0.4088$ was taken from the Drude model in Eq. (11). The numerical studies were performed with the discretization distances $h_{x}=0.1 \mu \mathrm{m}$ and $h_{y}=0.01 \mu \mathrm{m}$ in the cross section. Figure 8 shows the field distribution of the $H_{x}$ component at the interface between the dielectric and the silver layers. First we neglected the losses of the silver (i.e., the imaginary part of $\epsilon_{m}$ was chosen to be zero). One can clearly see a repetition of the input field at the self-imaging distance of $L_{\mathrm{si}} \approx 15.8 \mu \mathrm{m}$. Next, the calculations were performed by taking $\mathfrak{I}\left(\epsilon_{m}\right)$ into account. The result is shown in Fig. 9. Obviously, losses are quite significant so that the field amplitude drops to low values before the first selfimaging plane.

As described in Section 4.A, the losses in the waveguide decrease with increasing wavelength. For this reason we analyzed the same structure but now at a wavelength of $0.9 \mu \mathrm{m}$; see Fig. 10 . As expected,

(a)

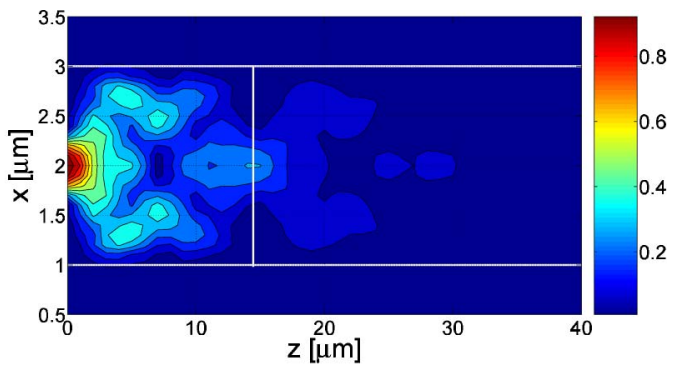

(b)

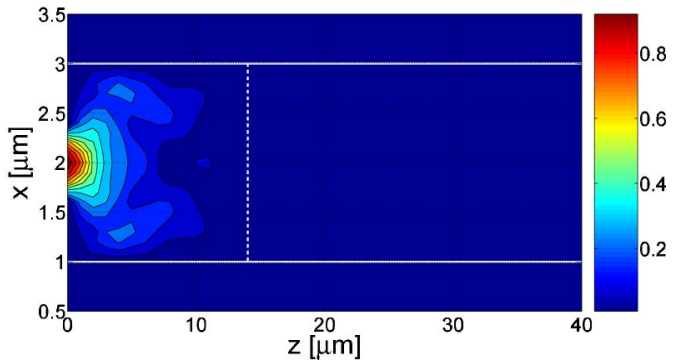

Fig. 11. (Color online) Field distribution of the $H_{x}$ component at the interface between the dielectric and the silver layer: (a) even excitation and ( b) odd excitation; parameters: $\lambda_{0}=0.6 \mu \mathrm{m}$ and $t=0.06 \mu \mathrm{m}$. 
Table 1. Self-Imaging Distances $L_{s i}$ Determined from the Field Distribution in Comparison with Eq. (17)

\begin{tabular}{lccccc}
\hline & $\lambda_{0}=0.6 \mu \mathrm{m}$ (even) & $\lambda_{0}=0.6 \mu \mathrm{m}$ (even) & $\lambda_{0}=0.9 \mu \mathrm{m}$ (odd) & $\lambda_{0}=0.6 \mu \mathrm{m}($ even$)$ & $\lambda_{0}=0.6 \mu \mathrm{m}($ odd) \\
\hline$t[\mu \mathrm{m}]$ & $0.1\left[\Im\left(\epsilon_{m}\right)=0\right]$ & 0.1 (lossy) & 0.1 (lossy) & 0.06 (lossy) & 0.06 (lossy) \\
$L_{\mathrm{si}}[\mu \mathrm{m}](\mathrm{MoL})$ & 15.8 & 14.5 & 9.1 & 15.0 & $($ field $\approx 0)$ \\
$L_{\mathrm{si}}[\mu \mathrm{m}][$ Eq. (17) $]$ & 15.0 & 15.0 & 9.15 & 14.24 & 15.84 \\
\hline
\end{tabular}

the decay of the field is more moderate and the selfimaging is more pronounced. Here it is important to note that the self-imaging distance is shorter than in the previous case. Although the wavelength occurs in the numerator of Eq. (17), the self-imaging distance decreases with $\lambda_{0}$ because $\Delta \bar{\beta}$ in the denominator is essentially proportional to $\lambda_{0}^{2}$. For $\lambda_{0}=0.9 \mu \mathrm{m}$ we find $L_{\mathrm{si}} \approx 9.1 \mu \mathrm{m}$.

From these considerations it becomes obvious that operating at larger wavelengths is recommended because of the lower attenuation of the field, at least with current devices. Figures 8 and 9 show field distributions for even modes and Fig. $1 \overline{0}$ for odd modes. The results for the respective other cases (odd fields for Figs. 8 and 9, even fields for Fig. 10) are not displayed because the even and the odd fields look similar in both cases. This is due to the large thickness of the metal layer, which, in agreement with the previous analysis, leads to the degeneracy of even and odd modes (see Fig. 5). For comparison we examine a situation in which one can expect a significant difference between even and odd modes. For our example we chose $t=0.06 \mu \mathrm{m}$ (see Fig. 5). The determined field distributions are shown in Fig. 11, which clearly indicates the difference. To quantify these results we compare the values for the losses from Fig. $\underline{5}$ with those determined from the simulations of the wave propagation. For the simulations the values for losses were derived from the maxima at $z=0$ and $z=L_{\mathrm{si}}$. For $t=0.06 \mu \mathrm{m}$ we calculated losses of 0.95 (odd case) and 0.71 (even case). These values were determined from the field distributions according to

$$
\operatorname{loss}_{1}=1-\left|H\left(z=L_{\mathrm{si}}\right)\right| /|H(z=0)| .
$$

We can compare these values with the loss factor of the eigenmodes shown in Fig. 5, from which we obtained 0.93 (odd case) and $0 . \overline{6} 9$ (even case). Here we used

$$
\operatorname{loss}_{2}=1-\exp \left\{-\bar{\alpha} k_{0} L_{\mathrm{si}}\right\} .
$$

Obviously, the numerical results are in good agreement. However, we must bear in mind that, in the first case [Eq. (18)], the complete field is determined by the superposition of the different modes that might not be exactly in phase. For this reason inequality $\operatorname{loss}_{1} \geq \operatorname{loss}_{2}$ holds.

Finally, we take a look at the self-imaging distances $L_{\mathrm{si}}$. For this purpose we compare the values obtained with Eq. (17) with those of the simulation, i.e., visual inspection of Figs. 8-11. In the latter case we looked for the maxima of the fields in the center of the waveguides. The results compiled in Table $\underline{1}$ show good agreement. Since the structure is lossy, the maximum field value depends on the interference of the modes and on their losses. Therefore, there are slight differences in the results obtained with Eq. (17). As one might expect, the best agreement is found between the MoL values and the ones from Eq. (17) when losses are neglected $(t=0.1 \mu \mathrm{m}$, $\lambda=0.6 \mu \mathrm{m}$ ). As one might also expect, $L_{\mathrm{si}}$ is different for the even and odd fields for $t=0.06 \mu \mathrm{m}$ $(\lambda=0.6 \mu \mathrm{m})$. For the odd modes the field decreases to zero before self-imaging can occur, therefore we did not assign a MoL value in Table $\underline{1}$ for this case.

\section{Summary}

We have examined the propagation of plasmonic waves in metallic waveguides. In particular, we considered self-imaging in plasmonic multimode waveguides. The analysis of an effect well known from classical optics and also from conventional dielectric waveguides lends itself to gain insight by drawing comparisons. We began our analysis with a study of the eigenmodes and examined the influence of the geometric waveguide parameters. This was done mainly with the purpose of investigating the attenuation properties of metallic waveguides. We found that losses can be reduced by decreasing the thickness of the waveguide, which in turn leads to an increased expansion of the field into the surrounding medium. Therefore, one observes the existence of a trade-off between loss and dimensional parameters. Furthermore, dependency exists between attenuation and wavelength: for longer wavelengths, attenuation is lower and the devices become more practical. Losses in plasmonic waveguides represent a general problem that needs to be solved before practical applications can be considered.

For now we can conclude from our study that the method of lines is capable of handling the material characteristics of metal structures to simulate plasmon wave propagation. The results obtained in the simulations are in good agreement with analytical predictions. In future work, we wish to develop configurations in which the losses are reduced, which might be achieved by structuring the metallic waveguides in a suitable manner. The use of exact simulation techniques can yield valuable results and insights for the experimental realization of such structured plasmonic waveguides. This can be combined with considerations of coupling electromagnetic fields to metallic waveguides.

\section{References}

1. H. Raether, Surface Plasmons, Vol. 111 of Springer Tracts in Modern Physics (Springer-Verlag, 1988). 
2. J. Ctyroky, J. Homola, P. Lambeck, S. Musa, H. Hoekstra, R. Harris, J. Wilkinson, B. Usievich, and N. Lyndin, "Theory and modelling of optical waveguide sensors utilising 20 surface plasmon resonance," Sens. Actuators B 54, 66-73 (1999).

3. R. D. Harris and J. S. Wilkinson, "Waveguide surface plasmon resonance sensors," Sens. Actuators B 29, 261-267 (1995).

4. Z. Liu, J. M. Steele, W. Srituravanich, Y. Pikus, C. Sun, and $\mathrm{X}$. Zhang, "Focusing surface plasmons with a plasmonic lens," Nano. Lett. 5, 1726-1729 (2005).

5. Z. Liu, J. M. Steele, H. Lee, and X. Zhang, "Tuning the focus of a plasmonic lens by the incident angle," Appl. Phys. Lett. 88, 171108 (2006).

6. M. R. Dennis, N. I. Zheludev, and F. J. G. de Abajo, "The plasmon Talbot effect," Opt. Express 15, 9692-9700 (2007).

7. R. Pregla and W. Pascher, "The method of lines," in Numerical Techniques for Microwave and Millimeter Wave Passive Structures, T. Itoh, ed. (Wiley, 1989), pp. 381-446.

8. R. Pregla, Analysis of Electromagnetic Fields and Waves: the Method of Lines (Wiley, 2008).

9. O. Conradi, S. Helfert, and R. Pregla, "Comprehensive modeling of vertical-cavity laser-diodes by the method of lines," IEEE J. Quantum Electron. 37, 928-935 (2001).

10. L. B. Soldano and E. C. M. Pennings, "Optical multi-mode interference devices based on self-imaging: principles and applications," J. Lightwave Technol. 13, 615-627 (1995).

11. H. F. Talbot, "Facts relating to optical science, No. IV," Philos. Mag. 9, 401-407 (1836).

12. A. W. Lohmann and D. A. Silva, "An interferometer based on the talbot effect," Opt. Commun. 2, 413-415 (1971).

13. J. Jahns, E. ElJoudi, D. Hagedorn, and S. Kinne, "Talbot interferometer as a time filter," Optik (Jena) 112, 295298 (2001).

14. O. Bryngdahl, "Image formation using self-imaging techniques,” J. Opt. Soc. Am. 63, 416-419 (1973).

15. R. Ulrich and G. Ankele, "Self-imaging in homogeneous planar optical waveguides," Appl. Phys. Lett. 27, 337-339 (1975).

16. S. F. Helfert, B. Huneke, and J. Jahns, "Self-imaging effect in multimode waveguides with longitudinal periodicity, J. Eur. Opt. Soc. 4, 09031 (2009).
17. S. F. Helfert and R. Pregla, "The method of lines: a versatile tool for the analysis of waveguide structures," Electromagnetics 22, 615-637 (2002),

18. R. Pregla and S. F. Helfert, "Modeling of microwave devices with the method of lines," in Recent Research Developments in Microwave Theory and Techniques, B. Beker and Y. Chen, eds. (Research Signpost, 2002), pp. 145-196.

19. H. J. W. M. Hoekstra, P. V. Lambeck, G. J. M. Krijnen, J. Čtyroký, M. D. Minicis, C. Sibilia, O. Conradi, S. Helfert, and R. Pregla, "A cost 240 benchmark test for beam propagation methods applied to an electrooptical modulator based on surface plasmons," J. Lightwave Technol. 16, 1921-1927 (1998).

20. M. Besbes, J. Hugonin, P. Lalanne, S. van Haver, O. Janssen, A. Nugrowati, M. Xu, S. Pereira, H. Urbach, A. van de Nes, P. Bienstman, G. Granet, A. Moreau, S. Helfert, M. Sukharev, T. Seideman, F. I. Baida, , B. Guizal, and D. V. Labeke, "Numerical analysis of a slit-groove diffraction problem," J. Eur. Opt. Soc. 2, 07022 (2007).

21. O. Conradi, S. Helfert, and R. Pregla, "Modification of the finite difference scheme for efficient analysis of thin lossy metal layers in optical devices," Opt. Quantum Electron. 30, 369373 (1998).

22. J. Gerdes, "Bidirectional eigenmode propagation analysis of optical waveguides based on method of lines," Electron. Lett. 30, 550-551 (1994).

23. S. F. Helfert, A. Barcz, and R. Pregla, "Three-dimensional vectorial analysis of waveguide structures with the method of lines," Opt. Quantum Electron. 35, 381-394 (2003).

24. R. Pregla, "Novel FD-BPM for optical waveguide structures with isotropic or anisotropic material," in Proceedings of the European Conference on Integrated Optics and Technical Exhibit (European Optical Society, 1999), pp. 55-58.

25. R. Pregla, "MoL-BPM method of lines based beam propagation method," in Methods for Modeling and Simulation of Guided-Wave Optoelectronic Devices (PIER 11), W. P. Huang, ed. (EMW Publishing, 1995), pp. 51-102.

26. P. Berini, "Plasmon-polariton waves guided by thin lossy metal films of finite width: bound modes of symmetric structures," Phys. Rev. B 61, 10484-10503 (2000).

27. J. J. Burke, G. I. Stegeman, and T. Tamir, "Surface-polaritonlike waves guided by thin, lossy metal films," Phys. Rev. B 33, 5186-5201 (1986). 